\title{
Parcel-Level Modeling to Analyze Transit Stop Location Changes
}

\author{
Peter G. Furth and Maaza C. Mekuria \\ Department of Civil and Environmental Engineering, \\ Northeastern University \\ Joseph L. SanClemente, Howard/Stein-Hudson Associates, Inc.
}

\begin{abstract}
Because of how important walk access is for transit travel, service changes that affect walking distance, such as route or stop relocation, call for modeling at a fine enough level to accurately reflect the often arbitrary aspects of the access network and of demand distribution within a zone. Case studies of stop relocation in Boston and Albany demonstrate the feasibility of parcel-level modeling on the unabridged street network using an assessor's database. Parcel-level demand is estimated by allocating observed on/off counts as a function of a parcel's land-use type, size (e.g., gross floor area), and location factors. With actual land-use and street network data, we show how stop service areas can deviate substantially from the simple geometric shapes that follow from assuming airline or rectilinear travel, and demand distribution can be far from uniform within a zone. These factors can significantly favor particular transit stop locations.
\end{abstract}

\section{Introduction}

Travel demand is typically modeled at the level of a traffic analysis zone. With improvements in computing power, zones have been getting smaller over the 
years. For automobile travel, zones are generally small enough that errors resulting from aggregating demand to the zonal level are insubstantial. However, for travel by transit, for which the access mode is usually walking, errors from representing an entire zone as having the same walking time can substantially distort an analysis.

We describe a new approach to modeling transit demand using individual land parcels, with walk access along the unabridged street network. This approach, which is roughly synonymous with assigning transit demand to every address, has become possible due to the growing local government use of geographic parcel-level databases for taxation and land-use planning, and the development of geographic information systems (GIS), GIS-based street maps, and GIS program utilities. We demonstrate this approach in stop-spacing case studies in Boston and Albany. Parcel-level modeling should also offer improved analysis for other applications sensitive to walk distance, including mode choice and transit route choice.

Modeling demand at the zone level forces one to assume either that demand is concentrated at a single point (zone centroid), or better yet, is distributed uniformly across the zone. The former is clearly unrealistic, and in many cases, demand is also far from uniform within a zone. Hospitals generate more transit demand than cemeteries, and apartment buildings more than single-family homes. Knowing each parcel's land use and size (not in land area, but in floor area or similar measure related to development intensity) allows one to distribute demand in a zone that naturally recognizes each parcel's trip generation and attraction potential.

GIS-based planning methods that account for land use have been developed for predicting demand along new transit routes (Gan, Liu, and Ubaka 2005) and along existing routes, using on-off count information (Bunner 2005). These approaches use block-level census data, greatly reducing aggregation error. However, they do not use the street network to determine walking paths or stop service areas.

With parcel-level modeling, the issue is not just "stop spacing" but "stop location." With demand distributed over the service area in a way that reflects development intensity, one can readily see the walking distance benefits of locating stops close to major generators and pockets of more intense development.

Zone-level modeling requires assumption of an ideal street network for walk access, which is estimated by such methods as airline distance multiplied by a circuity factor. However, street networks often have arbitrary barriers and discon- 
tinuities, diagonals, curves, and other features that affect walking distance. Modeling demand at the parcel level, using the actual street network for walk access, allows one to determine walk distance without making idealistic assumptions.

With idealistic assumptions about the street network, a transit stop's service area has simple boundaries-each stop's service area borders only those of its neighboring stops, with nice straight shed lines. We show that with realistic networks, service area boundaries can be far more complex, making some stop locations have much larger service areas than others, even if stops are equally spaced. This can affect optimal stop location; for example, adding a stop may have little benefit if that stop has a small service area.

In our application, parcel-level demand estimates are not synthesized directly from parcel attributes; rather, demand estimation begins with on-off counts, with parcel attributes used to distribute demand, mediated by a network analysis that determines which parcels lie in each stop's service area. Naturally, this logic can only be applied along existing routes, and for service changes that are not expected to change demand considerably-which is exactly the case for stop spacing. For such applications, basing demand on historic on-off counts makes the model self-calibrating, a great advantage. For applications to areas currently unserved by transit, or for which service changes are expected to result in large demand changes, parcel-leveling modeling would require the development of parcel-level transit-trip generation models.

\section{Walking Paths and Bus Stop Shed Lines}

As a simplification, walking distance to bus stops is often estimated based on airline distance, sometimes multiplied by a circuity factor. When the access network is a grid, a better assumption is rectilinear travel, meaning the access path consists of segments that are either parallel to or perpendicular to the bus route. In continuum models used in theoretical stop-spacing analyses, the rectilinear approach requires assuming an infinitely dense rectilinear access grid (Wirasinghe and Ghoneim 1981). When stop locations are given, one need not assume an infinitely dense grid; however, one must assume that (1) the route follows a straight line; (2) the streets in the access network form a rectilinear grid; (3) streets perpendicular to the bus route run without interruption across the full width of the service area; and (4) bus stops are all located at four-way intersections. This "ideal" urban layout was the justification for assuming rectilinear travel in our earlier work on stop spacing (Furth and Rahbee 2000). 
With idealized access paths, the shed line or service area boundary between adjacent stops is simply the perpendicular bisector of the segment joining the stops. Where the route is straight, or little enough curved so that adjacent shed lines do not intersect within the route's service area, a corollary of using perpendicular bisectors is that a stop's service area will border only that of its immediate upstream and downstream stops.

A more sophisticated way of determining shed lines, presented in Furth and Rahbee (2000), is based on minimizing not just walking time, but a weighted sum of walking and riding time. With this logic a traveler located halfway between two stops will not be indifferent, but will prefer the downstream stop. That shifts shed lines slightly upstream for boardings, and downstream for alightings, by an amount that depends on the ratio of the walking and riding speeds and the ratio of the walking and riding disutilties. Shed lines also vary by direction of travel, as travelers living midway between two stops will walk toward one stop when traveling in one direction, and toward the other when traveling in the other direction.

In practice, streets grids surrounding a bus route often deviate from the ideal conditions described before, and routes can curve or turn. As a result, shed lines are not as simple as (possibly shifted) perpendicular bisectors, and stop service areas can be considerably smaller or larger than one would otherwise expect. These considerations point to the value of using the street network, not simple geometric shapes, to determine stop service areas.

\section{Assigning Parcels to Stops}

With parcel-level modeling, a shortest path algorithm is applied on the street network to find the closest stop to each parcel. Shed lines are simply a result of this assignment. To account for the more sophisticated approach of minimizing a sum of walking and riding time, the assignment of parcel $k$ to a stop is found by first finding shortest path walking distances from parcel $k$ to every stop. (In practice, this step can be limited to stops within a certain practical distance of parcel $k$.) Then, for trips beginning at parcel $k$, the stop chosen is the one that minimizes, over all stops $i$,

$$
c_{\text {walk }} * d_{k i} / u_{\text {walk }}+\text { runTime }
$$

where:

$d_{k i} \quad$ equals (walking) distance from parcel $k$ to stop $i$

$c_{\text {walk }}$ is the cost of a minute of walking time relative to a minute of riding time (commonly given a value between 1 and 2.5) 
$u_{\text {walk }}$ is walking speed

runTime. equals running time from stop $i$ to the downstream end of the line

For trips ending at parcel $k$, the stop chosen is the one that minimizes

$$
c_{\text {walk }} * d_{k i} / u_{\text {walk }}+\text { runTime }_{i}
$$

where:

runTime $_{i} \quad$ equals running time from the upstream end of the line to stop $i$ In general, a parcel has four different assignments to stops, one each for inboundboarding, inbound-alighting, outbound-boarding, and outbound-alighting; similarly, stops have different service areas for those four combinations. (If inbound and outbound stops are colocated, and if running times in opposite directions are symmetric, the inbound-boarding and outbound-alighting service areas will be identical, as will the opposite pair.)

\section{Service Area Boundaries}

To illustrate how in a real network shed lines can differ from the simple shapes one would expect with straight-line travel, observe in Figure 1 the assignment of parcels to stops (stars) on a small section of Boston's B-line, a branch of the Green line light rail, for boarding passengers traveling inbound (in the figure, upward and to the right). The symbol of a parcel centroid is unique to the stop to which it is assigned, allowing one to see stop service areas. Shortest path trees are also shown, which allow one to verify the walking paths determined by the GIS "closest facility" utility used. The service area of Mt. Hood Road (identified as 9 in the figure) is quite unusual-it includes only two parcels on the north side of the transit line, largely because of the absence of an intersecting street on that side of the line. On the south side of Mt. Hood's service area, the shed line is shifted upstream toward Sutherland Road (identified as 10 in the figure).

In Figure 2, applying to outbound (leftward) travel in the same corridor, the difference in a stop's service area for boarding versus alighting can be seen. Stops are shown as stars. Parcel centroids are shown with different symbols according to their outbound alighting stop, while the manually drawn shed lines indicate service areas for outbound boardings. The boarding shed lines are all shifted upstream (toward the right), and alightings shed lines shifted downstream. This analysis emphasizes the need to determine separate service areas for a stop's ons and offs, as well as for each direction of travel. 


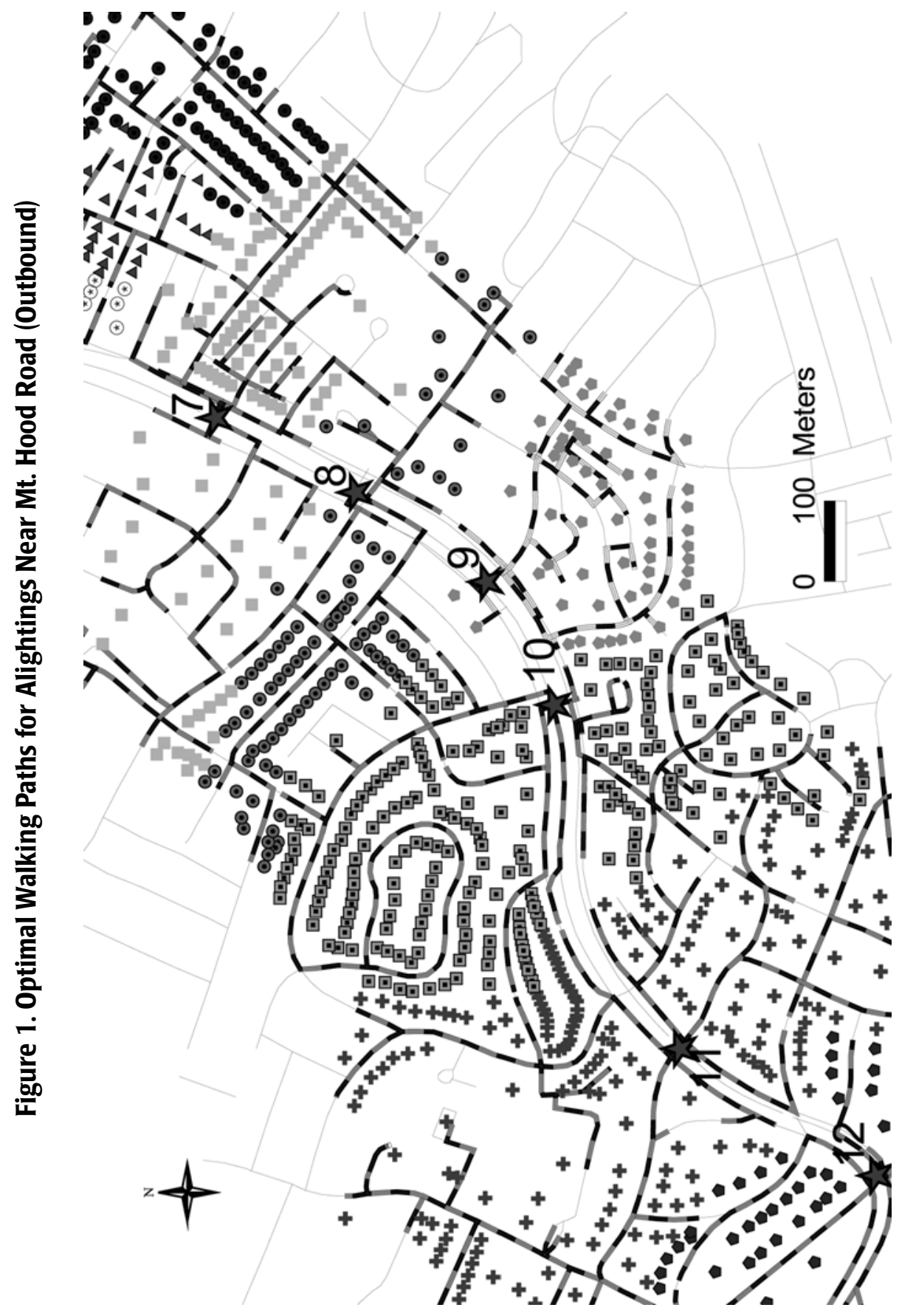




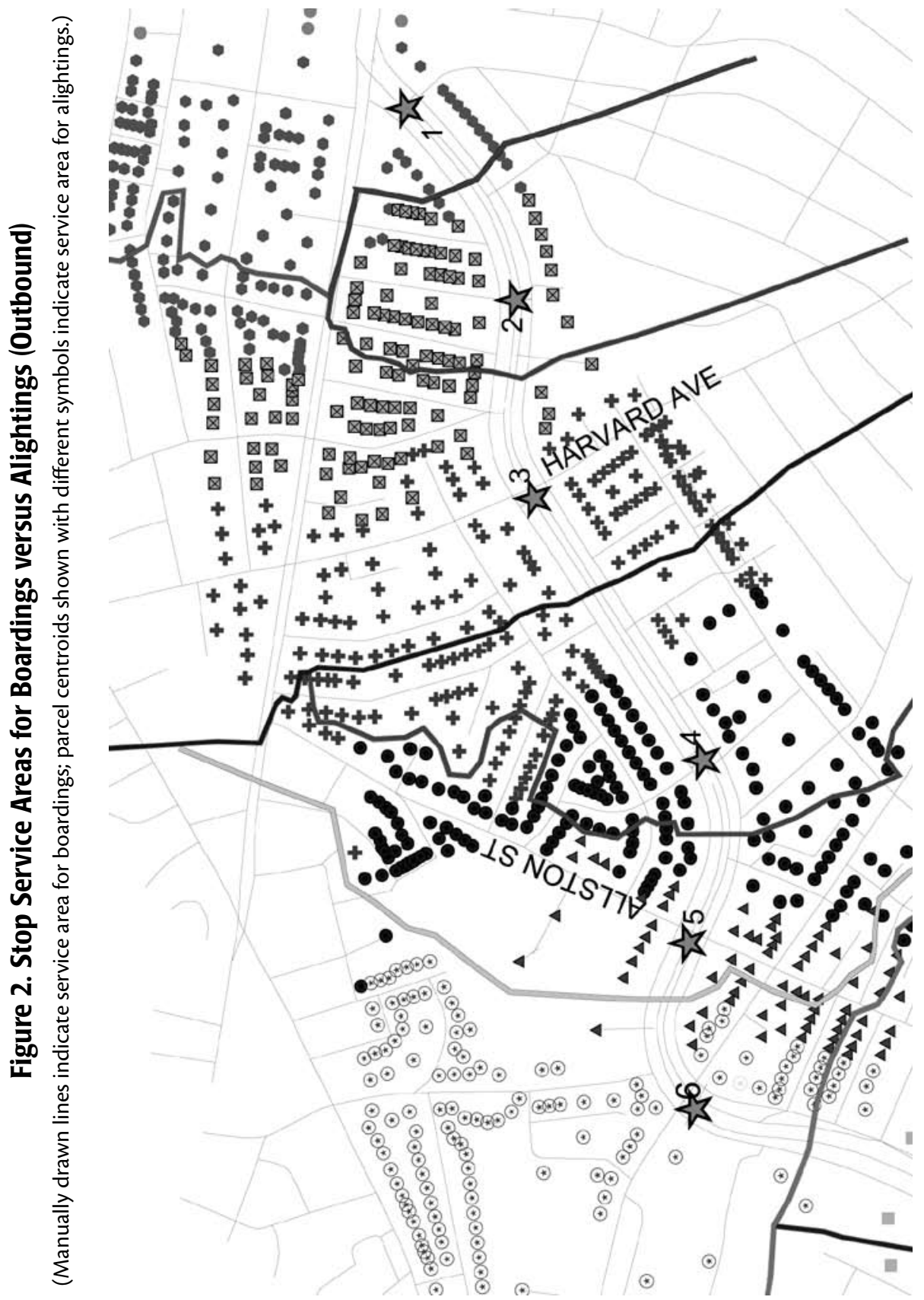


Figure 2 indicates how common it is for a stop's service area to border more than just those of its upstream and downstream neighbors. Due to curves in the transit line as well as irregularities in the access network, 9 of the 16 stops have service areas that border those of at least three other stops. The Summit Ave. and Griggs St. stops, located shortly before and after an S-curve, have outbound alightings service areas that border those of 4 other stops. It is also rather startling to see how many shed lines have segments that are more parallel than perpendicular to the transit line, due to discontinuities in the access network.

As this example shows, stop service areas can be influenced considerably by arbitrary aspects of the street network, pointing to the value of modeling demand on a scale smaller than a city block-ideally, a parcel-and modeling walking along the street network itself.

\section{Determining Parcel-Level Demand}

The initial goal in parcel-level transit demand modeling is to estimate the current number of trips originating and ending at each parcel. The most reliable approach skips traditional trip-generation and mode split steps, and instead directly uses on/off counts, which, after adjusting for possible passenger transfers, specify the trip generation within the stop's service area. All that remains then is to distribute the demand observed at the stops over each of the parcels in each stop's service area.

\section{Distributing Demand Over Parcels}

Reflecting the demand counted at a stop back to the parcels within its service area is a many-to-one trip distribution problem. Productions (trip origins, corresponding to "on" counts at a stop) are distributed separately from attractions (trip destinations, corresponding to "offs"). For productions, the general procedure is to determine for each parcel $k$ in a stop's service area a production strength onStrength $_{k^{\prime}}$ and to distribute demand in proportion to onStrength. A parcel's onStrength depends on two inherent characteristics, its land-use type and a measure of its size, called its size attribute, as well as two location characteristics:

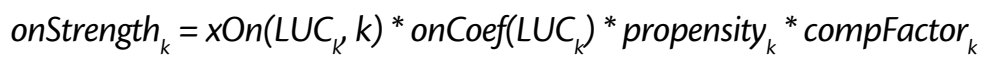

where:

$L U C_{k} \quad$ equals parcel $k$ 's land-use code or land-use type 
$x \operatorname{On}\left(L U C_{k^{\prime}} k\right)$ is the value of parcel $k^{\prime} s$ size attribute (a size attribute is specified for each land-use code)

onCoef $\left(L U C_{k}\right)$ is the coefficient that applies to a particular land-use code's size attribute

The variables propensity ${ }_{k}$ and compFactor ${ }_{k}$ are associated with parcel $k^{\prime}$ s location; they will be explained later.

The size attribute and coefficient are best explained with examples. For each landuse type, a single size attribute is chosen from among the attributes found in the land-use database. For the land-use type "single-family home," the size attribute used in the Boston case study was "living area," and so if $L U C_{k}$ was "single-family residential," $x O n\left(L U C_{k} k\right)$ was that parcel's living area, in thousands of square feet. In the Albany case study, however, the land-use database included the population in each residential parcel; therefore, for residential parcels in Albany, $x O n\left(L U C_{K}\right.$ $k$ ) was parcel $k$ 's population. For most nonresidential land-uses, $x O n\left(L U C_{k}, k\right)$ was "gross floor area" in the Boston case study, and "number of employees" in the Albany study. Other size attributes could be used if available such as "number of seats" for restaurant parcels.

A parallel procedure applies to distributing off counts. The resulting demand at each parcel may be tiny (a parcel may be allocated 0.06 trip origins and 0.09 trip destinations), but that is entirely appropriate for determining aggregate results such as change in demand or walking impact in response to relocating a stop or a route.

The demand that is distributed over parcels should exclude any transferring passengers, and requires that on-off counts distinguish transferring from nontransferring passengers. Walking impacts to demand arising from transfers is readily calculated based on the distance between the transfer stops.

\section{Estimating Trip-Generation Coefficients}

The coefficients onCoef(LUC) and offCoef(LUC) are trip-generation coefficients, reflecting the power of a land-use type to produce and attract transit trips per unit of the size attribute. One of the challenges in proving the practicality of this modeling approach was determining coefficients for different land-use types and size attributes. The Institute of Transportation Engineers (ITE) publication Trip Generation (1997) offers a wealth of trip-generation coefficients, mostly based on suburban developments with little or no transit access. ITE trip rates are available by time of day. In general, we used as coefficients the closest possible ITE coef- 
ficient, multiplied by "fraction entering" for on coefficients and "fraction exiting" for off coefficients, and by a factor indicating the likely transit share for a land-use type. Transit share data, in the form of mode share by trip purpose and time of day, was obtained from Boston's regional planning agency. The Boston mode share data showed that in the morning peak, the transit share for trips originating at homes was almost double that of trips originating elsewhere. Therefore, for the morning peak, ITE trip rates for residential parcel types were doubled relative to nonresidential parcel types. Some expert judgment was also used to estimate transit shares; for example, we assigned a high transit share to high schools and a low transit share to elementary schools.

Where land-use codes in a the parcel database encompass several ITE categories, ITE rates in the constituent categories were averaged, weighted by a (subjective) estimate of the relative presence of each category in the area. Where ITE rates used a different size attribute than the parcel data, they were adjusted by the ratio of the means of the size attributes, with mean values found in various demographic or land-use databases. The coefficients used in the Boston study are given in Table 1.

While this method of determining trip-generation coefficients is admittedly crude, we believe that they are adequate for most transit planning applications. Even crude rates accomplish the objective: assigning a stop's demand to the part of its service area where development is most intense, and away from where there is little development. Incorrect rates might mean, for example, that one block is assigned too much demand and another block in the same stop's service area is assigned too little. Because the rates are used for trip distribution, not trip generation, they should be transferable to other cities.

\section{Propensity and Competition Factors}

A few studies, summarized in Kittleson \& Associates et al. (2003), have shown that transit demand decreases at greater distance from a stop. Equation 3 includes the term propensity $k^{\prime}$, which can be used to indicate a greater or smaller propensity to make a transit trip based on distance from the closest stop. A simple propensity function, often used in gravity models, is exponential:

$$
\text { propensity }_{k}=\exp \left(-b d_{k}\right)
$$

where:

$d_{k} \quad$ equals distance from parcel $k$ to the nearest stop

$b \quad$ is a calibration parameter 


\section{Table 1. Trip Generation Coefficients}

\begin{tabular}{|c|c|c|c|c|c|c|c|}
\hline \multirow[b]{3}{*}{ Land-Use Code } & \multicolumn{7}{|c|}{ Production/Attraction Factors $^{1}$} \\
\hline & \multirow[b]{2}{*}{ Size Attribute } & \multicolumn{2}{|c|}{ AM Peak } & \multicolumn{2}{|c|}{ PM Peak } & \multicolumn{2}{|c|}{ Sat Midday } \\
\hline & & $\underset{\text { Coef. }}{\text { On }}$ & $\begin{array}{c}\text { Off } \\
\text { Coef. }\end{array}$ & $\begin{array}{c}\text { On } \\
\text { Coef. }\end{array}$ & $\begin{array}{c}\text { Off } \\
\text { Coef. }\end{array}$ & $\begin{array}{c}\text { On } \\
\text { Coef. }\end{array}$ & $\begin{array}{c}\text { Off } \\
\text { Coef. }\end{array}$ \\
\hline \multirow[t]{3}{*}{ R1-Residential 1-family } & HH member & 0.018 & 0.008 & 0.025 & 0.011 & 0.016 & 0.018 \\
\hline & 1000 sq. ft. LA & 0.036 & 0.012 & 0.023 & 0.041 & 0.028 & 0.033 \\
\hline & Unit & 0.070 & 0.023 & 0.045 & 0.081 & 0.054 & 0.063 \\
\hline \multirow[t]{3}{*}{ R2-Residential 2-family } & HH member & 0.021 & 0.009 & 0.025 & 0.015 & 0.017 & 0.019 \\
\hline & 1000 sq. ft. LA & 0.038 & 0.013 & 0.025 & 0.043 & 0.029 & 0.033 \\
\hline & Unit & 0.071 & 0.024 & 0.046 & 0.080 & 0.053 & 0.062 \\
\hline \multirow[t]{3}{*}{ R3-Residential 3-family } & HH member & 0.030 & 0.012 & 0.022 & 0.034 & 0.018 & 0.018 \\
\hline & 1000 sq. ft. LA & 0.042 & 0.016 & 0.027 & 0.043 & 0.027 & 0.027 \\
\hline & Unit & 0.056 & 0.022 & 0.037 & 0.057 & 0.036 & 0.036 \\
\hline \multirow[t]{3}{*}{ R4-Residential 4-6-family } & HH member & 0.030 & 0.012 & 0.022 & 0.034 & 0.018 & 0.018 \\
\hline & 1000 sq. ft. LA & 0.047 & 0.018 & 0.030 & 0.047 & 0.030 & 0.030 \\
\hline & Unit & 0.056 & 0.022 & 0.037 & 0.057 & 0.036 & 0.036 \\
\hline \multirow[t]{3}{*}{ A-Residential 7+ -family } & HH member & 0.035 & 0.013 & 0.025 & 0.039 & 0.021 & 0.021 \\
\hline & 1000 sq. ft. LA & 0.054 & 0.020 & 0.035 & 0.055 & 0.035 & 0.035 \\
\hline & Unit & 0.065 & 0.025 & 0.042 & 0.065 & 0.042 & 0.042 \\
\hline \multirow[t]{3}{*}{$\mathrm{CM}-$ Condominium } & HH member & 0.029 & 0.006 & 0.016 & 0.023 & 0.018 & 0.019 \\
\hline & 1000 sq. ft. LA & 0.051 & 0.010 & 0.029 & 0.040 & 0.031 & 0.033 \\
\hline & Unit & 0.065 & 0.014 & 0.037 & 0.051 & 0.039 & 0.042 \\
\hline Tax exempt land uses & 1000 sq. ft GFA & 0.097 & 0.244 & 0.230 & 0.108 & 0.052 & 0.065 \\
\hline Commercial land uses & $1000 \mathrm{sq} \mathrm{ft}$. GFA & 0.399 & 0.483 & 0.609 & 0.900 & 0.552 & 0.726 \\
\hline Mixed residential-commercial & $1000 \mathrm{sq} \mathrm{ft}$. GFA & 0.042 & 0.030 & 0.052 & 0.088 & 0.045 & 0.056 \\
\hline \multirow[t]{2}{*}{ Industrial land uses } & $1000 \mathrm{sq} \mathrm{ft}$. GFA & 0.013 & 0.061 & 0.056 & 0.020 & 0.008 & 0.008 \\
\hline & Acre & 0.141 & 0.539 & 0.448 & 0.268 & 0.125 & 0.122 \\
\hline
\end{tabular}

${ }^{1} \mathrm{LA}=$ living area; GFA = gross floor area.

A value of $b=0$ means propensity does not fall with distance. In our case studies, we arbitrarily used $b=0.0037 / \mathrm{m}$, for which transit-use propensity is three times greater for a parcel $100 \mathrm{~m}$ from a stop than for an otherwise equivalent parcel that is $400 \mathrm{~m}$ from a stop.

Traditionally, the phenomenon of decreasing demand with distance is treated as a simple step function: propensity is 1 out to a certain distance from the route (often 0.25 miles in bus route studies), and 0 after that. An exponentially decreasing propensity certainly seems logically superior to such an abrupt change. Of course, as distance from a route increases, one often arrives in the service area of another route, bringing up the issue of route competition. 
Competition from other transit routes in part of a stop's service area should logically lead to less demand than otherwise expected for the route of interest coming from that part of the service area. Ideally, the parcel level approach should be extended to include route choice models that account for walking distance as well as other route attributes such as waiting time and speed. For our application, we used a much simpler way of accounting for route competition: simply including in equation 3 a competition factor whose default value is 1 , and that can be set to a smaller value in parts of the route's service area to reflect the fraction of transit demand in that part of the service area that is drawn away to other transit lines, based on expert judgment.

Another possible extension would be to assign to each parcel a specific walking speed or unit walking cost. Special values could then be given to elderly housing and hospitals that would have the effect of making such parcels more sensitive to walking distance, giving them more weight in an optimal stop location problem.

\section{An Example}

Trip-generation results on a section of Boston's B-line are shown in Figure 3. Symbol size reflects the demand attracted by a parcel for outbound afternoon peak travel. Several items are evident. First, the results are consistent with on/off counts, showing heavy demand around stops with high off counts such as Harvard Ave. and Warren St. Second, they reflect development density. For example, Harvard Ave. has more intense development (apartment buildings) than other nearby streets, and so its parcels are assigned heavy demand relative to other nearby parcels. Third, one can see the effect of the exponential propensity function used, with parcel demand declining as one moves farther from the route.

\section{Application Results}

An example application to Boston's B-line is presented in Tables 2 and 3. Table 2 shows impacts by stop and overall for the base case (historical set of stops) for the section of the B-line between Packard's Corner (halfway to downtown) and Boston College (outer end of the line). Table 3 shows the change in impacts when one stop, Mt. Hood, is eliminated. The stop elimination affects only the neighboring stops; overall, walking time went up while riding time and operating cost went down. For the unit costs we used, the net impact was a savings of $\$ 35$ per hour, or $\$ 26,500$ per year for a three-hour weekday period. 


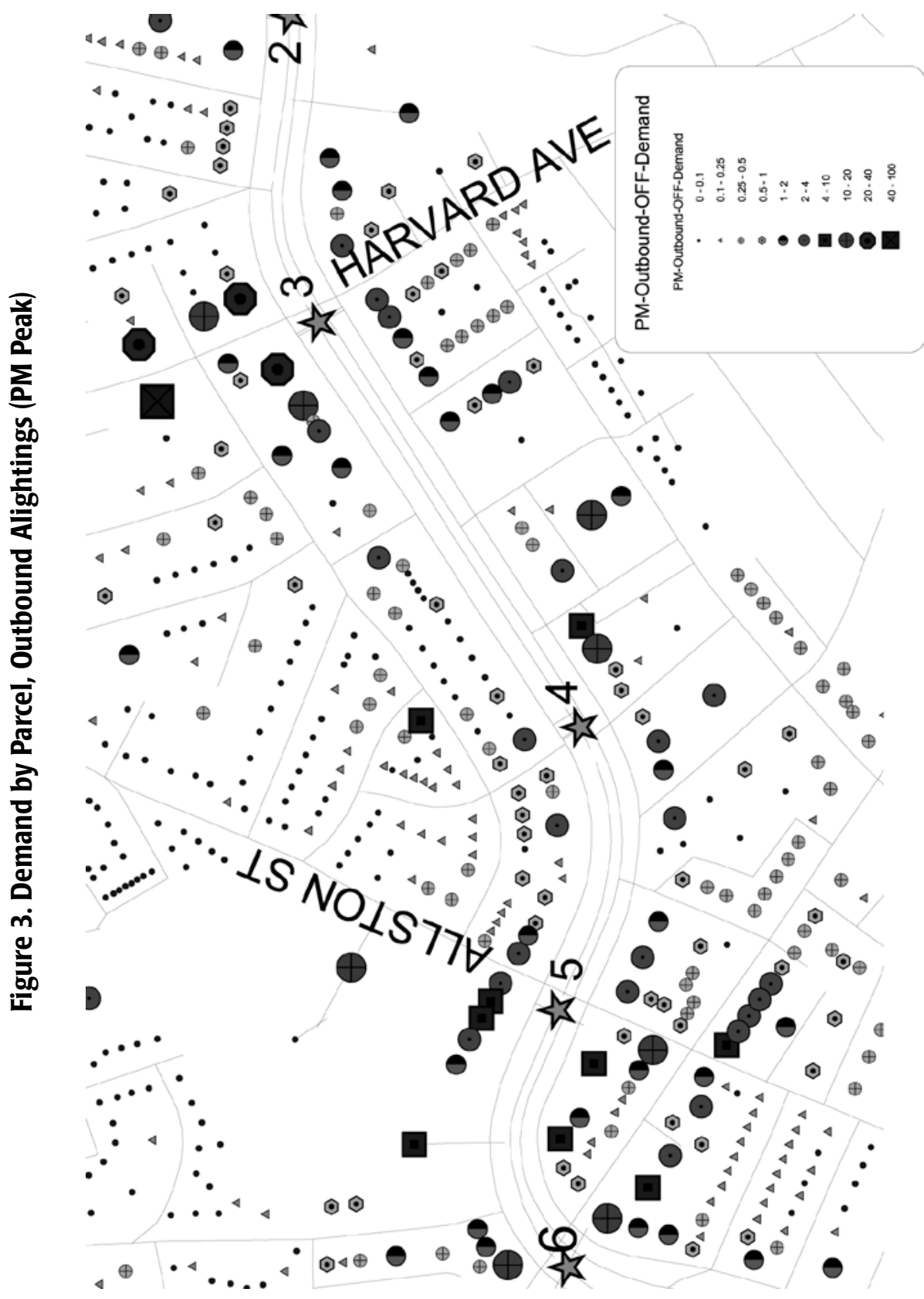


Journal of Public Transportation, Vol. 10, No. 2, 2007

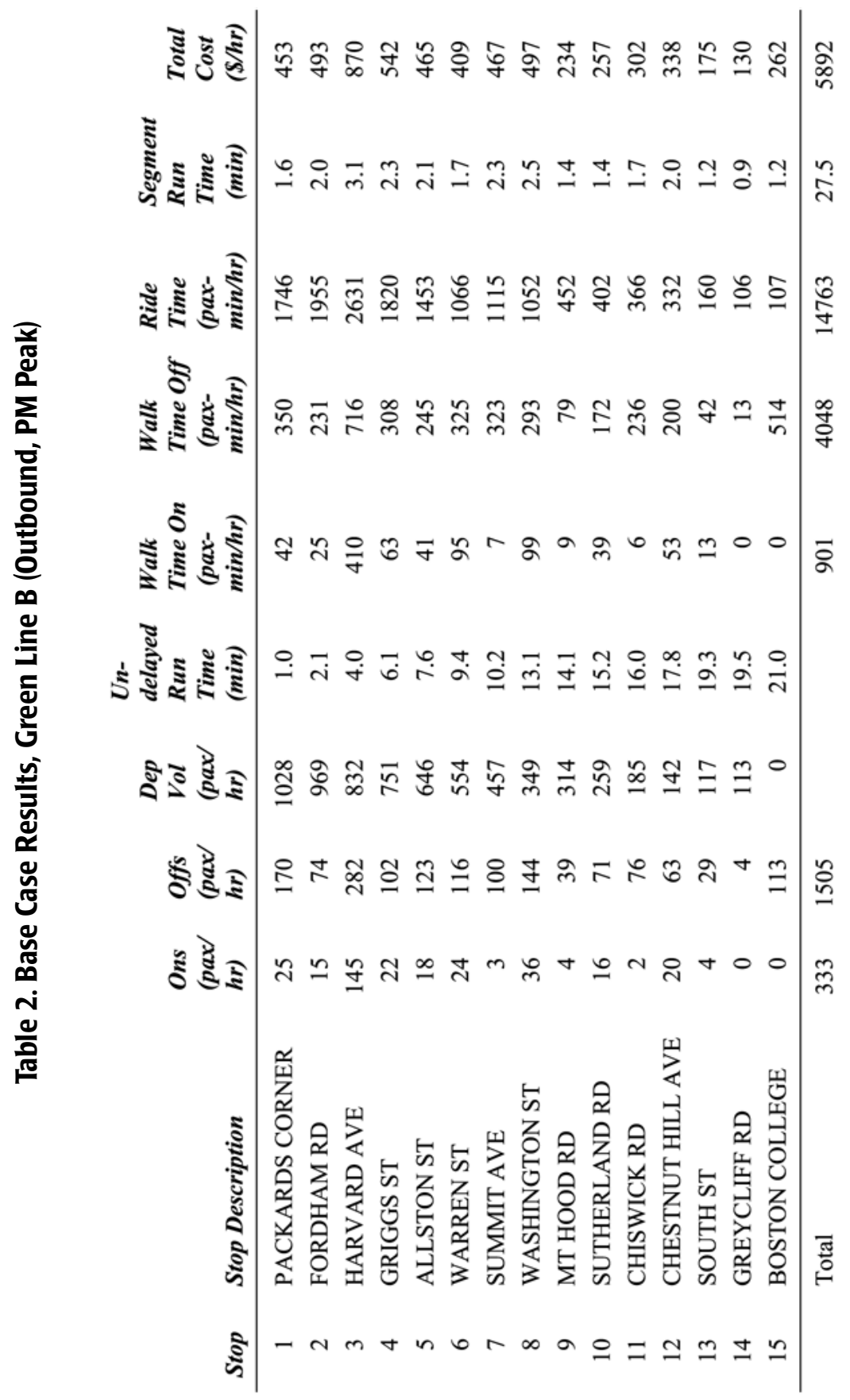




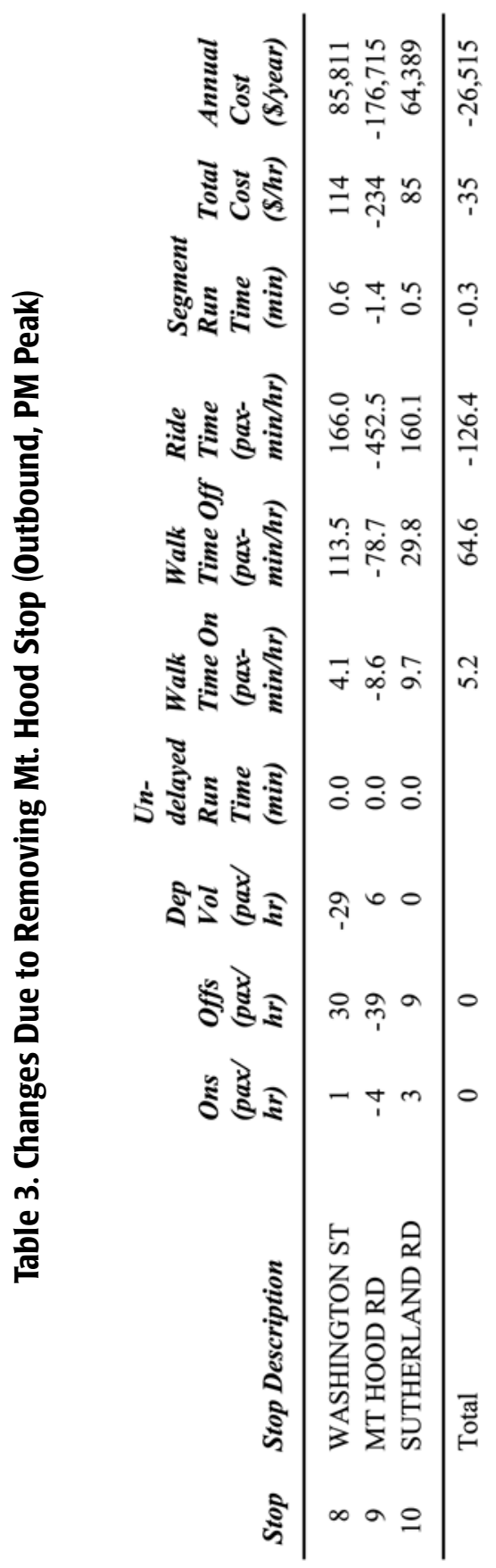


An interesting graphic produced in the study is shown in Figure 4, which illuminates the impact of eliminating the Mt. Hood stop. It shows walking paths to the nearest stop for parcels that formerly used Mt. Hood, and indicates how much each parcel's walking time has increased. One can see how small the impact is: eight parcels see their walking time increase by two to three minutes; all others have smaller increases.

\section{Practical Issues}

Implementing this new approach to transit demand modeling involves several practical issues.

Parcel-level databases are often restricted to a particular political jurisdiction. When a service area touches more than one jurisdiction, problems in securing and coordinating multiple databases arise. Also, some jurisdictions are less willing than others to share parcel data.

We found it necessary to edit street networks manually to ensure that they yielded reasonable walking paths. We had to add a few links to permit pedestrian crossings to some median stations where there are crosswalks that do not appear on the street map. We also deleted some alleys because their inclusion was forcing some parcels to make circuitous walking paths. The latter problem arose because the software utility that connects parcel centroids to the nearest link sometimes connected a parcel to the alleys at its rear rather than to the street at its front. Ideally, centroid connectors should be provided to both the street and the alley, allowing the shortest path routine to choose the better path.

The concept of passengers' walking "cost" can be expanded. Grade could be accounted for if the relevant data is included in the base map file. Other enhancements include accounting for streets segments that lack sidewalks or present safety challenges, and including pedestrian delay at street crossings.

Finally, the automation process was quite complex, involving numerous steps and intermediate databases. As is commonly known, GIS is a data-intensive process and hence processing large amounts of data in an efficient manner is required. Our work was greatly aided by two utilities available on the GIS platform we used: centroid creation and connection (used to convert parcels from polygons to points), and nearest facility (used to find walking paths from parcels to stops). 


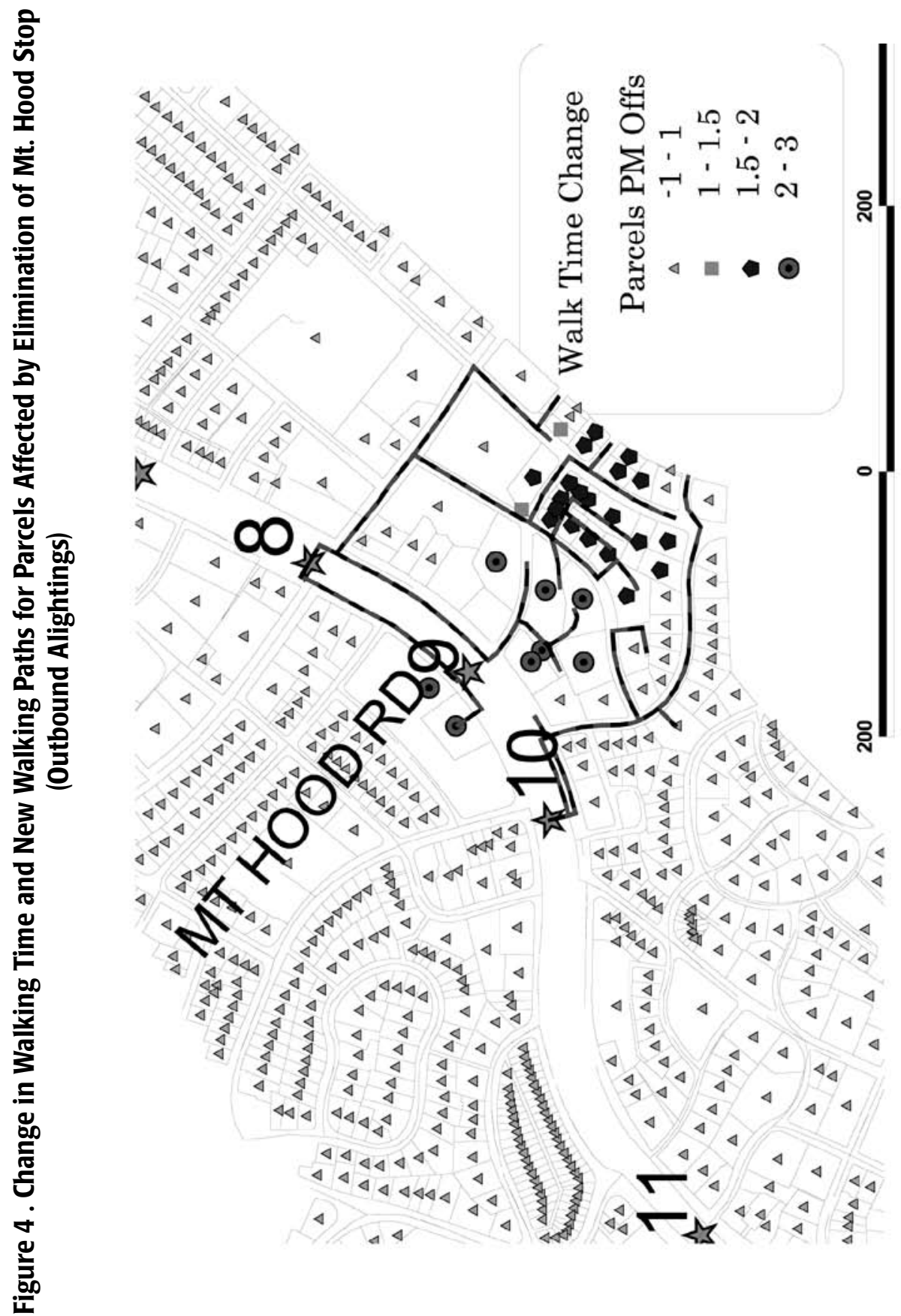




\section{Conclusions}

Modeling transit demand at the parcel level offers an improved way of accounting for walk access, one of the major user costs involved in transit travel. Using available parcel-level databases and street network data, it is possible to determine walking distance from each parcel to its closest stop in a way that accounts for irregularities and discontinuities in the street network. Using parcel-level data available from tax assessors and regional planning agencies, it is possible to distribute measured demand over the parcels in a stop's service area in a way that reflects differences in land use and intensity. With demand thus assigned to individual parcels, impacts of changing stop location can be determined, as demonstrated in two case studies. We believe that parcel-level modeling also offers promise for other transit planning applications in which walking distance plays an important role.

\section{Acknowledgement}

This research was supported by TCRP's Transit-IDEA program.

\section{References}

Bunner, R. T-Best. 2005. A comprehensive GIS-based stop-level transit boarding estimation and simulation tool. GIS-in-Transit Conference, Center for Urban Transportation Research, Tampa.

Furth P. G., and A. B. Rahbee. 2000. Optimal bus stop spacing through dynamic programming and geographic modeling. Transportation Research Record 1731: 15-22.

Gan, Albert, Kaiyu Liu, and Ike Ubaka. 2005. Florida transit geographic information system (FTGIS). GIS-in-Transit Conference, Center for Urban Transportation Research, Tampa.

Institute of Transportation Engineers. 1997. Trip generation, 6th ed. Washington, DC.

Kittleson \& Associates et al. 2003. Transit capacity and quality of service manual, 2nd ed., TCRP Report 100.

Wirasinghe, S. C., and N. S. Ghoneim. 1981. Spacing of bus stops for many to many travel demand. Transportation Science 15: 210-221. 


\section{About the Authors}

Peter G. FURTh (pfurth@coeneu.edu) is professor and chair of the Department Civil and Environmental Engineering at Northeastern University. He received BSCE (1977), MSCE (1980), and PhD in transportation systems (1981) from MIT. He is member of the American Society of Civil Engineers and Institute of Transportation Engineers. Dr. Furth's areas of research interests span traffic signal control, transit signal priority, transit operations modeling, and transit data collection and sampling.

MAAZA C.MeKURIA (mmekuria@coe.neu.edu) is a Ph.D. candidate at Northeastern University. He received BSCE from College of Engineering (1984), Gunidy, Chennai, India, and MSCE from Northeastern University (1991). His research interests include network systems, transit operations, GIS applications in transportation, traffic signal design, and data analysis.

JOSEPH SANCLEMENTE (jsanclemente@hshassoc.com) is a transportation engineer at Howard/Stein-Hudson Associates, Inc. He received a bachelor of science in civil engineering from Northeastern University and a master of science in civil engineering from Northeastern University. His areas of expertise include multimodal transportation planning, transit-oriented developments, and demand forecasting. 